\title{
Metodología aplicada: una estrategia de diseño para generar ambientes universitarios saludables
}

\author{
I. Palacios ${ }^{1}$ - E. Benítez² -D. Gómez ${ }^{3}$
}

\begin{abstract}
RESUMEN
Este artículo tiene como objetivo dar a conocer una investigación académica con relación a la importancia de promover y generar estrategias, así como acciones de generación y aplicación del conocimiento en congruencia al desarrollo académico integral de las comunidades universitarias, asociadas a los espacios en donde conviven, estudian, trabajan e interactúan cotidianamente. Por otro lado, presentar una metodología para identificar problemáticas específicas con relación a la percepción espacial y salud integral de las comunidades universitarias en los espacios abiertos, áreas verdes, áreas de convivencia y jardines. La presente investigación responde a observaciones de los organismos acreditadores, una de las cuales plantea la realización de planes de mejora a estos espacios. Con los resultados obtenidos se plantearan estrategias de solución con enfoque sustentable para contribuir al desarrollo de una vida saludable en las comunidades universitarias.
\end{abstract}

\begin{abstract}
The objective of this article is to present an academic research related to the importance of promoting and generating strategies, as well as actions of generation and application of knowledge in congruence with the integral academic development of the university communities, associated with the spaces where they live study, work and interact on a daily basis. On the other hand, present a methodology to identify specific problems related to the spatial perception and integral health of the university communities in the open spaces, green areas, areas of coexistence and gardens. The present investigation responds to the observations of the accrediting bodies, one of which proposes the realization of improvement plans for these spaces. With the results obtained, solution strategies with a sustainable approach will be pro-

posed to contribute to the development of a healthy life in the university communities.
\end{abstract}

Palabras Clave: Percepción espacial, salud integral, ambientes saludables, rendimiento académico, huella ecológica.

Keywords: Spatial perception, integral health, healthy environments, academic performance, ecological footprint.

\footnotetext{
1 Arquitecta, Maestría en Restauración Arquitectónica de bienes culturales por el Instituto de Antropología de la Universidad Veracruzana (México), Maestría en Arquitectura de Paisaje por el Colegio de Postgraduados Campus Córdoba (México), Coordinadora del CA Arquitectura y Hábitat Sustentable de la Facultad de Arquitectura Córdoba. Correo: irpalacios@uv.mx

2 Arquitecta, Maestría en Diseño Arquitectónico y Bioclimatismo por la Universidad Cristóbal Colón (México), Integrante del CA Arquitectura y Hábitat Sustentable.Correo: ebenitez@uv.mx

3 Arquitecto, Doctor en Arquitectura por la Universidad Politécnica de Madrid. (España), Integrante del CA Arquitectura y Hábitat Sustentable.Correo: dangomez@uv.mx
} 
Introducción

$\amalg$ s importante poner atención al diseño de ambientes que generen bienestar físico y emocional en los espacios universitarios, que son lugares en donde los alumnos, docentes y personal técnico manual, pasan jornadas de trabajo largas y requieren espacios de recreación multisensoriales, que ayuden a generar un mejor rendimiento en sus actividades cotidianas. Dado que es evidente la falta de opciones; es necesario hacer conciencia y propuestas, para generar ambientes universitarios saludables, con acciones socialmente responsables dirigidas a las comunidades universitarias, que propicien la salud, el bienestar y la productividad de las personas y que reduzcan el estrés en las actividades cotidianas. El diseño de espacios en manera estratégica ayuda a controlar el estrés en cada uno de los usuarios tales como: docentes, alumnos, administrativos, personal técnico y manual. Los integrantes de las comunidades universitarias que hacen uso cotidiano de estos espacios abiertos y cerrados, interactúan directa o indirectamente con los elementos sensoriales y perceptuales; por eso se debe propiciar un diseño integral y dinámico que interactué de manera armónica y equilibrada incluso en los espacios existentes, dándoles una reorganización, en donde el diseño o rediseño sea un recurso para el aprendizaje; pensado y creado para esta finalidad.

Un entorno saludable debe ser considerado como un espacio que Ofrece a una población protección contra las amenazas de su deterioro, Latapí (2015); dentro del Plan Nacional de Desarrollo está contemplado el Plan Sectorial de Salud, incluyendo los programas de acción 2013 - 2018 y el Programa de Entornos y Comunidades Saludables, con la única finalidad de lograr entornos favorables a la salud. La SMAES ${ }^{4}$ indica que una ciudad saludable debe contar con: áreas verdes estratégicamente diseñadas, con áreas de trabajo integradas y bien ubicadas con cordones o barreras verdes que protejan todas las áreas donde se desarrollan las actividades de sus habitantes. Y se puntua- liza que estos espacios deben brindar: bienestar, seguridad, conectividad, orden, accesibilidad universal, proporción y escala.

Dadas estas condicionantes referidas por Latapí (2015) es pertinente "ubicar" nuestras comunidades universitarias para poderlas diagnosticar bajo estos aspectos y en dado caso que no cumplan con todas las condiciones, promover y trabajar en propuestas de diseño paisajístico, ambiental, arquitectónico y urbano, para lograr que estas entidades académicas sean espacios estratégicamente saludables, aportando bienestar a la sociedad. Así también Lacomba (2013) menciona que entre los aspectos que hacen a una universidad sustentable se debe fomentar y trabajar en el cuidado del entorno construido con la arquitectura bioclimática, y sugiere implementar estrategias de diseño en las cuales se incluya investigaciones sobre ciudades sustentables y trabajos conjuntos con estudiantes.

Estos estudios estarán enfocados en: diseño bioclimático, diseño urbano de bajo impacto ambiental, estudios de estrategias por región, desarrollo de programas y diseños de acuerdo con el clima de cada región, programas por computadora para: calcular balance térmico y el sombreado en fachadas. Así como también estudios de ganancia por radiación solar en diferentes materiales, estudios sobre arquitectura de paisaje y cordones vegetales para ciudades, parques y jardines. Parte de estos aspectos han sido considerados de manera integral para este proyecto, el cual se desarrolla por etapas; en esta primera etapa se pretende plantear la metodología que fue diseñada y aplicada para la identificación de las problemáticas.

El proyecto planteado con base a lo identificado deberá mejorar directamente la calidad del aire, una reducción significativa del nivel de estrés, disminución de las ECNT ${ }^{5}$ así como de las enfermedades asociadas a estas, mitigación de impactos ambientales y suscitar patrones de vida saludables. Latapí (2015) indica que con una distribución adecuada de áreas verdes en condiciones favorables y agradables se logran estos beneficios en una comunidad o es-

4 SMAES se refiere a la Sociedad Mexicana de Arquitectos Especializados en Salud. Latapí (2014, 2015).

5 ECNT significa Enfermedades crónicas no contagiosas. Latapí (2015). 
pacio arquitectónico y urbano.

\section{Antecedentes del proyecto}

El Cuerpo Académico Arquitectura y Hábitat Sustentable de la Facultad de Arquitectura de la Universidad Veracruzana Región Orizaba Córdoba, trabaja en la línea de investigación denominada "Gestión Urbano Arquitectónica para un Desarrollo Sustentable", la cual aborda la temática con un enfoque interdisciplinario y multidisciplinario que observa, analiza y estudia la importancia del hábitat.

El grupo de investigación busca generar estrategias e instrumentos que ayuden a resolver problemas reales de diseño espacial y territorial, con una gestión del conocimiento socialmente responsable e intercambio de saberes a través de redes que sirvan para vincular la investigación con la docencia, así como también orientados al aprendizaje, la generación y la aplicación del conocimiento.

De acuerdo a este objetivo el cuerpo académico inició con la primera etapa del proyecto denominado: "Estrategias de Diseño para Generar Ambientes Universitarios Saludables".

En esta etapa, se plantea una metodología para diagnosticar cinco entidades académicas que se ubican en la Zona Metropolitana Córdoba - Orizaba; y con los resultados generar el planteamiento de los proyectos estratégicos de solución aplicando criterios de diseño paisajístico para espacios abiertos y de convivencia, áreas verdes, jardines y áreas comunes de conexión.

La pregunta es ¿por qué diseñarlos con este enfoque? Principalmente porque es necesario comprender en su verdadera magnitud el significado y los atributos de los espacios abiertos, sean jardines, áreas verdes o áreas comunes; que interactúan con la arquitectura existente. Porque al comprender por medio de un impacto benéfico en la percepción, al cual un espacio abierto está ligado; de forma ineludible con lleva a una serie de actividades saludables, tan solo esto contribuye directamente al proceso formativo de quien lo usa.

Por eso teniendo espacios construidos que correspondan a un lenguaje y carácter definido, como en el caso de las comunidades universitarias, se dará un proceso de aprendizaje integral de mayor rendimiento.

Dado esto es pertinente y necesario visualizar ese lenguaje simbólico y contextual influyente en la formación e interacción de los usuarios, porque precisamente estos ambientes pueden estimular la creatividad e incrementar el rendimiento académico de los alumnos; que mediante los espacios específicamente diseñados y reorganizados pueden ayudar a desarrollar competencias específicas en cada una de las disciplinas.

Algunas de las competencias genéricas a desarrollar mediante este tipo de espacios pueden ser:

Estimulación de los sentidos perceptuales; competencia que muchas disciplinas requieren como una habilidad o destreza que necesitan estimular en algunas experiencias educativas.

- Motivación a acciones de solidaridad y cuidado de la naturaleza; con esto se contribuye para lograr una universidad sustentable de manera integral.

- Observar e investigar; habilidad que todas las disciplinas requieren.

- Estimulación de la creatividad e incremento de la producción académica: competencia importante en el rendimiento académico y desempeño docente.

Esto contribuye a mejorar estilos y formas de aprendizaje porque definir espacios con características y atributos específicos invitan al usuario a interactuar en él. Esto hace que el usuario logre sentirse identificado y apropie el espacio como suyo para: pensar, dibujar, escribir, meditar, leer, relajarse y desarrollar actividades académicas más creativas y dinámicas.

Por lo tanto es importante referir las consideraciones de Rivera (2014) que menciona a los parques como indicadores de calidad de vida, símbolos de bienestar y espacios de uso recreativo. Creados por motivos ambientales y como recursos paisajísticos, los cuales favorecen a las actividades de ocio sin repercusión de bienestar; si bien los espacios abiertos, jardines 
y áreas verdes de las entidades académicas no son considerados como parques, si son espacios con las mismas características genéricas; solo cambia el término pero no la función principal. Dado esto se pueden considerar como indicadores de calidad de vida dentro de una comunidad universitaria.

\section{Marco teórico conceptual}

Es importante contextualizar y definir los conceptos que dan dirección al planteamiento metodológico en esta etapa de la investigación.

Salud integral abarca el nivel físico, mental y social, con plena satisfacción de necesidades y aspiraciones que propicien la calidad de vida según refiere la Carta de Ottawa para la promoción de la salud según menciona Latapí (2015).

Percepción espacial es una capacidad del ser humano de tener conciencia de su relación con el entorno en el espacio que lo rodea así como un proceso que construye representaciones sobre nuestro espacio por medio de los sentidos Pallasmaa (2012). Para su análisis es necesario considerar las experiencias multisensoriales, memoria e imaginación asociados a los espacios en donde se encuentra la población objetivo de análisis con esa interacción.

Diseño de ambientes saludables refiere a plantear o rediseñar espacios en entorno físico que promuevan salud, ocio y bienestar, seguridad, interacción social, movilidad, espacios seguros y accesibles. Se deben considerar también: índices de contaminación ambiental, acústica, visual y humos; aumentar espacios verdes de ocio y actividad física; resaltar los valores culturales distintivos mediante el urbanismo y promover actividades que fomenten la creatividad, que ayuden a contribuir a la prosperidad de la comunidad; según refiere el programa de "Ciudades Saludables" mencionado por Latapí (2015).

Felicidad es importante considerar este con- cepto tan subjetivo en este caso; porque está asociado a 3 componentes que se pueden considerar como constantes de la investigación: la esperanza de vida, la percepción de bienestar y la huella ecológica; así lo muestra Latapí (2015) en la cartografía que refleja el resultado que emite el Happy planet index (2013); por lo tanto al diseñar el instrumento metodológico es importante su consideración. Aunque es un concepto subjetivo si se considera como cuantificable en relación a la salud integral y a la percepción espacial.

Estrategias para lograr una salud integral referidas en la Carta de Ottawa: 1. Elaboración de una política pública sana (Definición de políticas públicas en todos los sectores y niveles); 2. Creación de ambientes favorables en los siguientes aspectos: Físico, social, económico, político, de recreación y cultural (Los ambientes de trabajo y recreativos deberán organizarse para generar condiciones de vida saludable, seguras y estimulantes). 3. Fortalecimiento de la acción comunitaria (Componente básico de la promoción de la salud; es la participación comunitaria en la definición de prioridades, toma de decisiones y acciones para mejorar sus condiciones); 4. El desarrollo de las aptitudes personales (Trabajo colaborativo de investigación entre profesionales y sociedad); 5. La reorientación de los servicios sanitarios (Hacia la investigación, la educación y la formación profesional; orientada hacia el respeto a la cultura de las personas favoreciendo las condiciones de vida y de salud en los diferentes tipos de comunidades sociales).

Con base a las 5 estrategias planteadas en la Carta de Ottawa para lograr una salud integral y el marco conceptual se plantean los objetivos que se quieren alcanzar con la metodología diseñada para el diagnóstico de las entidades académicas seleccionadas. El muestreo que se realiza con este instrumento es la primera aproximación a la identificación del problema, considerándola como una radiografía general que nos permita comprobar la existencia y magnitud del problema. Considerando tener una segunda etapa para analizar el fenómeno a detalle. 
Desarrollo de la investigación aplicada:

Se plantea una metodología para diagnosticar cualquier género de edificio; en este caso se aplicó al género destinado a la educación pública superior para determinar sus problemáticas de salud ligadas a los espacios abiertos, de convivencia, áreas verdes y jardines. Teniendo la aplicación en 5 entidades académicas de la Universidad Veracruzana ubicadas en la Zona Metropolitana Córdoba - Orizaba; Facultad de Arquitectura Córdoba, Facultad de Ciencias Químicas Orizaba, Facultad de Enfermería Orizaba, Centro de Idiomas Córdoba, y Facultad de Medicina de Ciudad Mendoza.

La metodología planteada está diseñada con el objetivo de: Obtener información para generar un diagnóstico y realizar un planteamiento estratégico de solución con relación a la percepción espacial y su relación con la salud integral directamente relacionada al tiempo de permanencia de los usuarios en los espacios donde desarrollan sus actividades cotidianas; lo cual está directamente ligado al proceso formativo de la población objetivo. Así como también detectar si el bienestar físico y emocional que un ambiente espacial genera en el usuario influye en el rendimiento académico y actividades cotidianas. Las constantes de investigación están definidas como: salud integral, percepción espacial, ambientes saludables y 3 componentes que se consideran en el concepto de felicidad: la esperanza de vida, la percepción de bienestar y la huella ecológica. Las variables de análisis son: el tiempo de permanecía en los espacios y las característica urbanas contextuales de cada entidad académica. Considerando estos elementos se diseñó la encuesta para el muestreo de exploración y aproximación al problema.

\section{Objetivos específicos:}

- Identificar el tiempo que pasa la población objetivo en los espacios.

- Conocer el sentido de percepción espacial de los usuarios en actividades cotidianas.
- Identificar los espacios con más problemas de percepción espacial y sensorial.

- Identificar si los usuarios perciben la problemática existente y ven pertinente una estrategia de diseño en los espacios abiertos, de convivencia, áreas verdes y jardines que ayuden a reducir su estrés y mejore sus actividades académicas.

Para lograr los objetivos se diseñó un instrumento para realizar un muestreo aleatorio de aproximadamente 10\% de la población objetivo de cada entidad académica que se analizó. La encuesta se aplicó durante el periodo escolar Agosto 2017 - Enero 2018 abarcando las 5 entidades académicas seleccionadas antes mencionadas, durante dos días consecutivos.

El instrumento encuesta está diseñado y estructurado de la siguiente manera:

- Datos generales de la entidad académica y de la población objeto: Sexo, edad, tipo de usuario (estudiante, docente, administrativo, personal técnico y manual).

- ¿Cuántas horas te encuentras en tu facultad? Se pusieron los siguientes rangos: 5 hrs, de 5 a 8 hrs, de 8 a 12 hrs y más de 12 hrs.

- ¿En qué espacios de tu facultad te encuentras más a gusto? En esta pregunta se puso un espacio con el plano de la facultad indicando con letras las áreas en donde el encuestado podía marcar los espacios.

- ¿Cómo es tu interacción con las áreas verdes de tu facultad? Con esta pregunta podemos saber si se usan o no las áreas verdes.

- ¿Consideras que los espacios abiertos de tu facultad son adecuados para realizar actividades como descansar, leer, meditar, concentrarte, relajarte, escribir, recreación? Al hacer esta cuestión sabemos la relación directa de las áreas a analizar con las actividades cotidianas y si están diseñadas específicamente para esas actividades.

- ¿Te gusta el diseño de tus áreas verdes, espacios abiertos, de convivencia y jar- 
dines? Determina la percepción espacial con relación a los aspectos estéticos y de diseño.

- ¿Crees que los espacios abiertos de tu facultad áreas verdes y jardines influyen en tu estado de ánimo? Esta pregunta ayuda a saber si al estar en un espacio hay variaciones en emociones asociadas a las actividades académicas o cotidianas de la población de estudio. Percepción espacial asociado a salud integral.

- ¿Qué tipo de contaminación percibes dentro de tu facultad: Auditiva, visual, ambiental olfativa, otras? Ayuda a identificar los elementos polisensoriales que están afectando o beneficiando un espacio abierto, áreas verdes, de convivencia o jardín.

Con base en los resultados de la aplicación del cuestionario y el análisis de información se identificaron las problemáticas y clasificaron de acuerdo a los objetivos específicos y datos que se esperaban identificar con claridad.

Dentro de la estructura metodológica es importante realizar una contextualización del objeto de estudio; orientado a identificar su potencial espacial y paisajístico así como problemáticas multisensoriales de percepción. Se aplicó para esta etapa la siguiente estructura:

Cuadro 1. Muestra los resultados obtenidos del muestreo en relación a los objetivos específicos.

\begin{tabular}{|c|c|c|c|c|}
\hline $\begin{array}{l}\text { POBLACION } \\
\text { OBJETIVO }\end{array}$ & \multicolumn{4}{|c|}{$\begin{array}{l}\text { Objetivos específicos a lograr mediante la aplicación de la encuesta y la estructura meto- } \\
\text { dológica planteada. } \\
\text { Los resultados obtenidos corresponden al muestreo aleatorio de aproximadamente un ran- } \\
\text { go de } 5 \text { a 10\% de la población de cada entidad académica diagnosticada en relación de la } \\
\text { matricula durante el periodo en que se aplicó la encuesta. Cabe destacar que es la primera } \\
\text { aproximación exploratoria para identificar la existencia del problema. } \\
\text { Conceptos asociados a los objetivos: Salud integral, percepción espacial, ambientes saluda- } \\
\text { bles en relación a la contaminación visual, ambiental, acústica, humos; y Felicidad relacio- } \\
\text { nada con percepción de bienestar, esperanza de vida y huella ecológica). }\end{array}$} \\
\hline $\begin{array}{l}\text { FACULTAD DE } \\
\text { APLICACIÓN Y } \\
\text { MUESTREO } \\
\text { (Aplicadas aleato- } \\
\text { riamente a: estu- } \\
\text { diantes, docentes, } \\
\text { personal adminis- } \\
\text { trativo, personal de } \\
\text { servicio) }\end{array}$ & $\begin{array}{l}\text { Obj. 1. Identificar el } \\
\text { tiempo que pasa la } \\
\text { población objetivo en } \\
\text { los espacios. } \\
\text { Se muestran los ran- } \\
\text { gos que obtuvieron } \\
\text { el mayor porcentaje. }\end{array}$ & $\begin{array}{l}\text { Obj. 2. Conocer el } \\
\text { sentido de percep- } \\
\text { ción espacial de los } \\
\text { usuarios en activida- } \\
\text { des cotidianas }\end{array}$ & $\begin{array}{l}\text { Obj. 3. Identificar } \\
\text { los espacios con más } \\
\text { problemas de per- } \\
\text { cepción espacial y } \\
\text { sensorial. Identifica- } \\
\text { ción de espacios me- } \\
\text { nos saludables. }\end{array}$ & $\begin{array}{l}\text { Obj. 4. Identificar si } \\
\text { los usuarios perci- } \\
\text { ben la problemática } \\
\text { existente y ven per- } \\
\text { tinente una estrate- } \\
\text { gia de diseño en los } \\
\text { espacios abiertos, de } \\
\text { convivencia, áreas } \\
\text { verdes y jardines que } \\
\text { ayuden a reducir su } \\
\text { estrés y mejore sus } \\
\text { actividades académi- } \\
\text { cas. }\end{array}$ \\
\hline $\begin{array}{l}\text { CENTRO DE } \\
\text { IDIOMAS } \\
\text { CORDOBA, VER } \\
73 \text { encuestas apli- } \\
\text { cadas }\end{array}$ & De 5 a 8 hrs & $\begin{array}{l}\text { Relación de interac- } \\
\text { ción con áreas ver- } \\
\text { des nulas. Espacios } \\
\text { estresantes e inse- } \\
\text { guros. No producen } \\
\text { ninguna sensación o } \\
\text { emoción. }\end{array}$ & $\begin{array}{l}\text { Las áreas verdes } \\
\text { tuvo el mayor por- } \\
\text { centaje }\end{array}$ & $\begin{array}{l}\text { Si lo requieren y ven } \\
\text { la importancia del } \\
\text { diseño en esos espa- } \\
\text { cios. }\end{array}$ \\
\hline $\begin{array}{l}\text { FACULTAD DE } \\
\text { CIENCIAS } \\
\text { QUIMICAS } \\
\text { ORIZABA,VER } \\
108 \text { encuestas apli- } \\
\text { cadas }\end{array}$ & De 8 a 12 hrs ó más & $\begin{array}{l}\text { Las áreas más } \\
\text { transcurridas son las } \\
\text { que generan mejor } \\
\text { estado de ánimo, } \\
\text { sin embargo no son } \\
\text { los más agradables. } \\
\text { Sensación de insegu- } \\
\text { ridad. }\end{array}$ & $\begin{array}{l}\text { Áreas de conviven- } \\
\text { cia y áreas verdes, } \\
\text { área de canchas de- } \\
\text { portivas }\end{array}$ & $\begin{array}{l}\text { Reflejan una preocu- } \\
\text { pación y necesidad } \\
\text { de que sus espacios } \\
\text { mejoren en imagen y } \\
\text { definición de activi- } \\
\text { dades para su uso. }\end{array}$ \\
\hline
\end{tabular}




\begin{tabular}{|c|c|c|c|c|}
\hline $\begin{array}{l}\text { FACULTAD DE } \\
\text { ARQUITECTURA } \\
\text { CORDOBA,VER } \\
50 \text { encuestas apli- } \\
\text { cadas }\end{array}$ & De 8 a 12 hrs ó más & $\begin{array}{l}\text { No hay interacción } \\
\text { con áreas verdes } \\
\text { grandes porque se } \\
\text { percibe inseguridad, } \\
\text { provocan estrés y } \\
\text { empeoran su estado } \\
\text { de ánimo. }\end{array}$ & $\begin{array}{l}\text { Áreas verdes, esta- } \\
\text { cionamiento y áreas } \\
\text { verdes sin uso. }\end{array}$ & $\begin{array}{l}\text { Si quisieran unas } \\
\text { áreas verdes y es- } \\
\text { pacios de descanso } \\
\text { y convivencia dise- } \\
\text { ñados, agradables y } \\
\text { confortables princi- } \\
\text { palmente en perio- } \\
\text { dos de entregas de } \\
\text { proyectos y exáme- } \\
\text { nes. } \\
\text { También se identi- } \\
\text { ficó el realizar un } \\
\text { plan de mejora en las } \\
\text { áreas verdes como } \\
\text { una observación de } \\
\text { organismos acredita- } \\
\text { dores. }\end{array}$ \\
\hline $\begin{array}{l}\text { FACULTAD DE } \\
\text { MEDICINA } \\
\text { CD.MENDOZA } \\
73 \text { encuestas apli- } \\
\text { cadas }\end{array}$ & De 8 a 12 hrs ó más & $\begin{array}{l}\text { Usan las áreas } \\
\text { verdes muy poco y } \\
\text { no son agradables. } \\
\text { Los espacios de } \\
\text { convivencia como } \\
\text { explanadas son las } \\
\text { concurridas pero no } \\
\text { son agradables. }\end{array}$ & $\begin{array}{l}\text { Áreas de conviven- } \\
\text { cia, áreas verdes y } \\
\text { jardín explanada }\end{array}$ & $\begin{array}{l}\mathrm{Si} \text { les gustaría un } \\
\text { cambio y un redise- } \\
\text { ño de sus áreas ver- } \\
\text { des y espacios abier- } \\
\text { tos. Principalmente } \\
\text { espacios para leer, } \\
\text { descansar y relajarse } \\
\text { un rato en periodos } \\
\text { de exámenes. }\end{array}$ \\
\hline $\begin{array}{l}\text { FACULTAD DE } \\
\text { ENFERMERIA } \\
\text { ORIZABA,VER } \\
100 \text { encuestas apli- } \\
\text { cadas }\end{array}$ & De 8 a 12 hrs & $\begin{array}{l}\text { Las áreas verdes son } \\
\text { las que les provocan } \\
\text { mayor estrés y no } \\
\text { son muy agradables } \\
\text { pasar largo tiempo } \\
\text { ahí. }\end{array}$ & $\begin{array}{l}\text { Áreas verdes y jardi- } \\
\text { nes, áreas de convi- } \\
\text { vencia }\end{array}$ & $\begin{array}{l}\text { Si quieren espacios } \\
\text { mejor diseñados y } \\
\text { con elementos defi- } \\
\text { nidos para realizar } \\
\text { actividades de lectu- } \\
\text { ra, descanso, relajan- } \\
\text { tes. }\end{array}$ \\
\hline
\end{tabular}

Fuente. Elaboración propia con base a las tesis que fueron producto de la colaboración de alumnos de la Facultad de Arquitectura Córdoba cursando la experiencia recepcional del periodo Agosto 2017 - Enero 2018; los cuales aplicaron el instrumento metodológico para la justificación de los proyectos ejecutivos que elaboraron. Colaboración con el CA Arquitectura y Hábitat Sustentable; con el objetivo de vincular: la investigación, la docencia, aprendizaje para la generación y aplicación del conocimiento.

Contextualización: En la etapa de contextualización se define la legibilidad urbana y espacial de la entidad académica seleccionada en la cual para contextualizar se aplica la metodología de Cabeza (1993) y Schjetnan (2004); en esta misma etapa de reconocimiento del lugar se realiza el diagnóstico de potencial paisajístico con base a los cuestionamientos planteados por Steinitz (1990) y el análisis polisensorial definido por Zarate (2004).
Con el diagnóstico y la contextualización se plantea el plan estratégico e integral y se aplican criterios de diseño paisajísticos sensoriales y terapéuticos para generar ambientes universitarios saludables atendiendo problemáticas identificadas puntualmente.

Y conduce el proyecto hacia un enfoque de sustentabilidad con una visión socialmente responsable.

Y como etapa final de esta estructura metodológica implementar un $\mathrm{POE}^{6}$ (Post ocupancy

6 POE término usado para realizar una evaluación post ocupacional (Post ocupancy evaluation). Es un instrumento que ayuda a medir las reacciones que un espacio produce en las personas después de ser construido, habitado u ocupado. 
evaluation) Es necesario porque relaciona acciones reales (vivir, sentir y tocar el espacio) de acuerdo a las posibilidades en las cuales se vaya desarrollando el proyecto.

El objetivo de usar un POE es considerar: la medición y registro de lo que paso en el espacio diseñado en tres momentos: espacio actual con problemáticas, espacio en proceso de transformación y cambio, espacio modificado al 100\% diseñado con base en la problemáticas identificadas). Prácticamente esta evaluación sistemática nos ayuda a verificar el objetivo planteado para los usuarios con respecto a su bienestar físico y emocional correlacionado con los espacios diseñados y su rendimiento académico en su proceso formativo. Esta etapa se puede llevar a cabo mediante la estrategia de urbanismo táctil.

\section{Conclusiones}

Las conclusiones de esta aproximación y exploración al problema de identificación de espacios no saludables son definidas por las contribuciones que se obtienen con la aplicación de la metodología planteada como estrategia académica de investigación y sistematización de un proceso, para conseguir la solución más creativa a una problemática existente, en este caso, en los edificios dedicados a la educación pública superior. También se podrá comprobar que este instrumento es aplicable a los proyectos de otros géneros arquitectónicos: hospitales, centros penitenciarios, edificios destinados a la educación pública básica y media superior; así como en distintas escalas sean de nivel internacional, nacional, estatal, regional, municipal y local. Para finalizar, se considera que con este proceso se contribuye a lo siguiente con relación a los objetivos que se pretendían alcanzar con la aplicación metodológica de diagnóstico:

Aportar datos importantes y de fuente directa de los usuarios es con el objetivo de generar estrategias de diseño y políticas pertinentes a las acciones y decisiones que se logren plantear para mejorar estos ambientes en las entidades académicas; que no cabe duda que son espacios que generan cambios en los usuarios que repercuten en sus actividades y rendimiento académico. Las principales problemáticas genéricas identificadas son:

- Las áreas que son más concurridas aunque no sean las más agradables son las que mejoran más el estado de ánimo de los usuarios. Las áreas verdes, espacios abiertos, espacios de convivencia y jardines resultaron ser los menos saludables y son los que tienen más problemas de percepción espacial en relación a sensaciones de inseguridad correlacionados a estados de ánimo, contaminación visual, ambiental, visual y acústica.

- En todas las entidades académicas diagnosticadas se identifica que no hay interacción directa con las áreas verdes y jardines; que la mayoría tiene una percepción de estar en un espacio inseguro y descuidado que les provoca estrés y desconfianza a ser usadas por periodos largos de tiempo.

- El descuido y la falta de diseño generan un límite entre el usuario y las áreas verdes, de convivencia y jardines.

- Las Facultades de Ciencias Químicas Orizaba, Arquitectura Córdoba y Medicina de Ciudad Mendoza son las que reflejan tener jornadas de permanencia más largos en los espacios; y expresan tener la necesidad de lugares con espacios más definidos y específicos para descansar, leer y trabajar de manera tranquila en sus proyectos. Se identifica un problema debido a las largas jornadas educativas, debido a que los horarios en algunos periodos son dispersos entre clase y clase. Estos periodos de tiempo son empleados para trabajar en los proyectos escolares, dentro de las instalaciones de la universidad, por lo que estos espacios se encuentran en constante uso pero carecen de los elementos necesarios para facilitar el desempeño académico.

- La población objetivo manifiesta que hay temporadas en que se sienten muy estresados por la carga de trabajo y no tienen un lugar especial para relajarse o esperar tiempos largos de una clase a otra ya que 
viven lejos y prefieren quedarse en la facultad para ahorrar un poco de dinero en el traslado. Y esto hace que usen los espacios existentes pero no produce en ellos un bienestar o algún cambio de ánimo; al contrario alguno de los espacios los estresan más.

- La carencia de áreas verdes adecuadas, para el esparcimiento y desarrollo creativo de los alumnos influye en el aumento de estrés y ansiedad principalmente durante los periodos de entregas parciales, finales y exámenes.

- Se identifica en todas las entidades alto potencial espacial en áreas verdes y espacios abiertos pero refieren que son los que tienen las vistas más desagradables y estresantes convirtiéndose en espacios no usados o usados pero con cierto desánimo.

- Como conclusión final es necesario remarcar que las acciones y planteamientos para realizar los proyectos de mejoramiento en los espacios abiertos, áreas verdes, espacios de convivencia y jardines se hicieron considerando las 4 problemáticas principales identificadas: a) largas jornadas de estancia y trabajo en las edificaciones, b) que las áreas más usadas y concurridas o fueron las más agradables y eran las que más cambios de ánimo provocaban en los usuarios, c) la desarticulación entre áreas verdes y espacios abiertos con relación al uso con la población objeto; y d) se identifica alto potencial espacial; para generar diseños paisajísticos y sensoriales que mitiguen los efectos del estrés cotidiano e incrementen los rendimientos académicos. Se espera contribuir al incremento de los niveles académicos y productivos de la comunidad universitaria; fomento y desarrollo de la sustentabilidad aplicada; desarrollo creativo mediante la estimulación de habilidades por medio de los espacios diseñados con un fin específico; las estrategias de diseño en la planeación de espacios con estas características; traerá beneficios en cambios de estilo de vida a la sociedad generando: entornos saludables y comunidades universitarias felices; las cuales sin lugar a duda serán más productivas y creativas.

\section{Referencias}

Cabeza, A. (1993). Elementos para el Diseño de Paisaje. México: Trillas.

Lacomba, R. (2013). La ciudad sustentable. Creación y rehabilitación de ciudades sustentables. México: Trillas.

Latapí, J. (2015). Anmm.org.mx.Publicaciones. Recuperado el 5 de Mayo de 2016, de Ciudades saludables en el contexto internacional. Libro en línea. CONACYT. http:// www.anmm.org.mx/publicaciones/CAnivANM150/ L26_ANM_Ciudades_saludables.pdf

Palacios, I. (2017). Establecimiento de criterios para diseñar un jardín sensorial. Tesina. Colegio de Postgraduados - Campus Córdoba.

Pallasmaa, J. (2006). Los ojos de la piel. La arquitectura y los sentidos. Barcelona: Gustavo Gili.

Rivera, M. (2014). Los parques urbanos como indicadores de calidad de vida símbolos de bienestar y espacios de uso recreativo. Universidad and Empresa, 16(27), 215237.

Schjetnan, M. (2004). Principios de diseño urbano-ambiental. México: Limusa. SEDESOL. (1992). Obtenido de http:// www.redicsa.org/ARQUITECTURA/SEDESOL\%201.pdf

Zarate, H. (s.f.). Manual para la modificación de senderos interpretativos en ecoturismo. Recuperado el 1 de junio de 2016 https://es.scribd.com/doc/80507872/Manual-Senderos-Interpretativos-mexico 